\title{
State of the Art on Alternative Methods to Animal Testing from an Industrial Point of View: Ready for Regulation?
}

\author{
Rachel Ashton ${ }^{1}$, Bart De Wever ${ }^{2}$, Horst W. Fuchs ${ }^{3}$, Marianna Gaca ${ }^{4}$, \\ Erin Hill $^{5}$, Cyrille Krul ${ }^{6}$, Albrecht Poth ${ }^{7}$ and Erwin L. Roggen ${ }^{8}$
}

${ }^{1}$ Ashton Editorial Consulting, London, UK; ${ }^{2}$ ALTEXA Development, Monte Carlo, Monaco; ${ }^{3}$ CellSystems, Biotechnologie Vertrieb GmbH, Troisdorf, Germany; ${ }^{4}$ British American Tobacco, G\&D Centre, Southampton, UK; ${ }^{5}$ IIVS, Gaithersburg, MD, USA; ${ }^{6}$ TNO, Healthy Living, Zeist, Netherlands; ${ }^{7}$ Harlan Cytotest Cell Research GmbH, Roßdorf, Germany; ${ }^{8}$ Novozymes AS, Bagsvaerd, Denmark

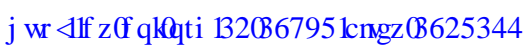

\section{Summary}

Despite changing attitudes towards animal testing and current legislation to protect experimental animals, the rate of animal experiments seems to have changed little in recent years. On May 15-16, 2013, the In Vitro Testing Industrial Platform (IVTIP) held an open meeting to discuss the state of the art in alternative methods, how companies have, can and will need to adapt and what drives and hinders regulatory acceptance and use. Several important points arose from the meeting. First, industry and regulatory bodies should not wait for complete suites of alternative tests to become available, but should begin working with methods available right now (e.g., mining of existing animal data to direct future studies, implementation of alternative tests wherever scientifically valid rather than continuing to rely on animal tests) in non-animal and animal integrated strategies to reduce the numbers of animals tested. Second, sharing of information (communication), harmonization and standardization (coordination), commitment and collaboration are all required to improve the quality and speed of validation, acceptance and implementation of tests. Finally, how alternative methods can be used in research and development before formal implementation in regulations should be considered. Here we present the conclusions on what can be done already and suggest some solutions and strategies for the future.

Keywords: non-animal testing, legislation, regulation, in vitro modeling, harmonization, standardization

\section{Highlights}

- In spite of partial replacement of methods in testing strategies, in no area can animal testing yet be completely replaced.

- Substantial progress is needed in the development of methods to replace, reduce and refine animal experiments (the 3Rs approach).

- The emphasis on mechanisms and modes of action will be crucial to minimize animal studies in toxicological evaluation.

- All stakeholders must not only promote the use of alternative tests, but also take responsibility to find pragmatic ways to change.

- Sharing of information, harmonization, standardization and collaboration will help to improve the quality of tests and speed of validation.

\section{Introduction}

Despite changing attitudes towards animal testing and striking advances in technology, substantial progress has been made in the development of alternative methods to achieve replacement, reduction and refinement of animal experiments (the 3Rs approach). Although the rate of animal testing varies between regions, little seems to have changed in recent years in Europe (EC, 2010). Legislation is in place that encourages the phasing out of animal testing (EU, 2010), yet some companies seem reluctant to move away from proven animal tests to new testing strategies. For the cosmetics industry animal testing and marketing of animal-tested products is now banned, but no validated assays are available to replace tests for most classic toxicological endpoints and kinetic data. Therefore, alternative approaches are needed to ensure safety, but the change needs to be much more strongly supported by all stakeholders. 
On May 15-16, 2013, the In Vitro Testing Industrial Platform (IVTIP) held an open meeting to discuss the state of the art in alternative methods, how companies have, can and should adapt and what helps and hinders regulatory validation. The meeting included perspectives from industry, regulators, contract research organizations and societies for the humane treatment of animals. Several key themes became clear. Here we present the conclusions on what can be done now and suggest some solutions and strategies for the future.

\section{Current status of legislation}

On July 11, 2013, the European Cosmetics Regulation (EC/1223/2009) fully replaced Council Directive 76/768/EEC, which was put in place in 1976 to ensure the free circulation of safe cosmetic products in the European internal market. The seventh amendment of the Directive in 2003 introduced the phasing out of animal testing and, eventually, marketing of products that have involved animal testing (CEC, 2003), for which the final deadline passed on March 11, 2013. Despite the lead-up period and the extension of animal testing for some complex endpoints, the European Commission acknowledges that the full replacement of animal testing by alternative methods for toxicokinetics, skin sensitization, repeated dose testing and reproductive toxicity is not yet possible (EC, 2013a). It has, however, decided to go ahead with the ban for several reasons: It was feared that further postponement of the ban or allowance of applications for dispensations on testing would not reflect the political preferences of the European Parliament and could "diminish determination to swiftly develop alternative test methods. Past experience demonstrates clearly that animal testing provisions in the cosmetics legislation have been a key accelerator in relation to the development of alternative methods and have sent a strong signal far beyond the cosmetics sector and far beyond Europe" (EC, 2013a). An impact assessment (EC, $2013 b$ ) showed that stakeholder opinions diverged on the degree of impact related to the ban coming into force as planned. Also, where animal tests were phased out in 2009 for which no alternatives were available, no major negative impacts have been seen so far (EC, 2013a). In all industries dealing with compounds and ingredients, legislation has been in place since 2010 (2010/63/EU) that requires companies to prioritize non-animal methods where they are available (EU, 2010). Bridging the gap between knowledge and legislative commitments by the innovative development of predictive models by in vitro assessment of pathways and mechanisms is, therefore, an urgent requirement.

\section{Alternative methods and approaches}

\subsection{Mechanisms and modes of action}

Although the study of mechanistic data is not new, the emphasis on mechanisms and modes of action in toxicological evaluation is a notable shift and will be crucial to minimization of animal studies. Knowledge of modes of action will provide information on key events from the molecular to the organism levels that can be applied to assessment of exposure risks and population respons- es. Several challenges, however, will need to be faced. First, it is unlikely that any single animal test will be replaced by a single alternative test, and multiple approaches will need to be applied to achieve the same results. Thus, rather than thinking about the quality of individual tests, researchers will have to start designing testing strategies that include several tests, which will take more planning and require more advanced validation assessments. Also, the risk that results will be obtained by chance due to increasing the number of tests, and ways to counteract those effects will need to be taken into account at the study design stage (e.g., sample size, significance threshold, etc.). Owing to the increase in the number of tests and the potential for high-throughput analyses, the volume of data will also be greatly increased. How to store, organize, present and interpret larger amounts of information in a transparent and useful way will need to be further explored and harmonized (Hardy et al., 2012). Not least of the challenges is how to open up industry and regulators to the changes in testing strategies. Modes of action as part of the 3Rs strategy are relatively newly acknowledged (Zuang et al., 2013) and there is a lot to learn. So far, the greatest amount of experience with alternative methods has been gained in the pharmaceutical industry. However, recently, the Safety Evaluation Ultimately Replacing Animal Testing (SEURAT-1), a Seventh Framework Programme project, jointly financed by the European Commission and the cosmetics industry, has been set up to assess the role of mechanistic and mode-of-action research in toxicology.

Adverse outcome pathways (AOPs) represent the sequential progression of events from exposure to outcome (Ankley et al., 2010). They are conceptual constructs of linkages between a direct molecular initiating event and adverse outcomes at different levels of biological organization and are, therefore, highly relevant to risk assessment. Information can be classified at the molecular, macro-molecular, cellular, organ, organism and populations levels. The aim is to identify ways in which normal processes are altered by exposure to stressors and whether or not these changes result in harmful outcomes. In theory the number of ways in which cells function should be finite, although the pathways by which alterations occur could be infinite. Although extrapolation of the results to the human population will take years, mapping of these toxic effects has begun to build the human toxome. The pathways concept represents a paradigm shift from the traditional safety evaluation of identification of a hazard, risk characterization and exposure assessment to predictive science (Leist et al., 2008).

Alternative methods for testing of skin sensitization, carcinogenicity, and repeated-dose and reproductive toxicities have also begun to be developed as part of broader testing strategies. Most of the key stages in skin sensitization AOPs can now be tested by laboratory in vitro tests with skin models, peptide reactivity assays, activation of innate response, epidermal equivalent potency testing and activation of dendritic cells. Some of these test methods have entered regulatory-driven validation studies and acceptance is foreseen in the near future. Additionally, where tests might have been limited to a few doses in animals, owing to cost, resource and legislative constraints, in vitro assays allow a wide range of doses to be tested. This should yield much more highly relevant information on (low) dose-response effects where in animal tests the minimum internal dose that 
causes an effect might be irrelevantly high compared with that required in humans (Crump, 2011).

The Organisation for Economic Co-operation and Development (OECD) has suggested that AOPs be recorded according to a standardized notation (OECD, 2013). It purports that the three basic elements for AOPs are molecular-initiating events, which describe how the chemical interacts with the initial biomolecule; intermediate events; and adverse outcomes, which should be specific and well-defined. Key issues in building AOPs are where to start (which pathway), how and where to limit them, how to assess and identify completeness and robustness, how to identify which uses will be appropriate and the validation to humans. The building of pathways can start from any of the three basic elements but, although adverse outcomes can result from multiple molecular initial events and vice versa, each AOP should be limited to a single molecular initial event and one adverse outcome. Information from different levels of biological organizations, however, can be integrated into a pathway. The OECD also provides guidance on the minimum conditions for evidence (Bradford-Hill criteria; Hill, 1965), annotation of the pathway, quality assessments, scope and how to make quantitative linkages.

AOPs already help to direct testing by enabling the linkage of events to outcomes or testing of predicted relations assessed in vitro. Thus they are likely to have multiple applications that can reduce the need for animal testing (Tab. 1). To help prediction, AOPs will need to show key actions that link events and how pathways intersect and interact and, therefore, creation of predictive paradigms is complex. This will hopefully become easier as more AOPs are created, developed and assessed, although information from a lot of AOPs will be needed. Thus an additional challenge will be improvement of the tools for and approaches to storage and access of AOPs to avoid duplication and ensure standardization.

\subsection{Emerging technologies and techniques}

Traditional toxicology animal testing relies heavily on dose tests followed by the detection and pathological evaluation of manifested toxic lesions. The so-called omics technologies, bioinformatics, systems biology and computation biology (Altenburger et al., 2012; Collings and Vaidya, 2008; Panagiotou and Taboureau, 2012) enable high-throughput analysis of treatment-related changes at the molecular level and, therefore, might provide a means for predicting toxicity before classic toxicological endpoints are seen.
The pillars of modern toxicology could be considered as organotypic cultures including "human-on-chip", omics (high-throughput studies, bioinformatics, bioengineering), pathways of toxicity (human toxome) and integrated testing strategies.

In the USA, the National Toxicology Program (http://ntp.niehs. nih.gov/) recognized that the technological advances in molecular biology and computer science offered an opportunity to use in vitro biochemical-based and cell-based assays and non-rodent animal models for toxicological testing. These assays allow for high throughput at a much reduced cost. In some assays, many thousands of chemicals can be tested simultaneously in days. The National Toxicology Program is collaborating with other federal organizations, the NIH Chemical Genomics Center and the National Center for Computational Toxicology, the Environmental Protection Agency and the Food and Drug Administration, to develop the Tox 21 project. It aims to develop, validate and translate innovative, high-throughput chemical screening methods to characterize key steps in toxicity pathways, identify mechanisms of action for further investigation and develop predictive models for in vivo biological response. Currently, more than 10,000 compounds $(8,100$ of which are unique substances) are being analyzed by a variety of automated methods. This enables high-throughput analysis to be undertaken day and night, which yields results much faster than could ever be achieved by human teams. At the meeting, it was purported that this approach will be the future of industry testing if the AOPs can be translated or linked to human diseases.

\subsection{Use of existing resources}

Irrespective of whether animal testing remains legal in certain industries or whether validated non-animal models are available, a huge amount of data have been collected from years of animal testing. The phasing out of animal experiments does not mean that these data become invalid. Rather, they comprise a valuable resource that should be perused carefully to help guide the development of non-animal alternative methods and prioritize future areas of study (Ekin, 2006; Hardy et al., 2012; Rebholz-Schuhmann et al., 2012; Schrage et al., 2011), particularly while alternative methods are awaiting approval. In vitro tests should not be validated by comparison with animal studies, which sometimes have limited predictive value compared with the human situation, and new thinking about the validity of new approaches must be adopted to ensure innovative approaches are approved.

\section{Tab. 1: Roles for adverse outcome pathways in reducing the need for animal testing}

Current and near-term uses of adverse outcome pathways:

- Inform chemical categories and structure activity relationships

- Identify hazards

- Prioritize chemicals for further assessment

- Support and enable interpretation of existing and new information

- Contribute to development of integrated testing strategies that maximize useful information gained from minimum testing

Possible future roles:

- Prioritization of a wider range chemicals for assessment

- Identification of key events for which non-animal tests can be developed to facilitate mechanism-based, non-animal chemical assessment

- Creation of predictive toxicological assessments with low uncertainty and high human relevance

- Eventually lead to the replacement of animal testing 
Mining of existing data obtained with technologies, such as metabolomics and transcriptomics, systems biology and bioinformatics, as well as new experiments, are helping to systematically organize knowledge of what happens from exposure to a toxicant to effects at the molecular, macromolecular, organism and population levels. This approach has played an important part in the Tox21 initiative. Existing data were provided with many of the compounds that were contributed to the project and have yielded important information to guide studies.

\subsection{The relationship between industry and regulators}

A substantial proportion of Member States do not yet adhere to 2010/63/ EU, which requires prioritization of non-animal tests over animal tests whenever possible. That IVTIP is concerned about compliance with this requirement was clearly represented at the meeting. The reasons underlying poor adherence to legislation are manifold and are seen at multiple levels. A key phrase in the legislation is "Member States shall ensure that a procedure is not carried out if another method or testing strategy for obtaining the result sought, not entailing the use of a live animal, is recognized under the legislation of the Union." Exactly what is classed as recognized, however, is unclear and interpretations seem to vary widely. Where to find clarifying information also seems unclear. Thus, the number of regulatory bodies and companies making substantial efforts to adopt alternative methods to animal testing might be reduced.

To temper this uncertainty, the relationship between industry and regulators needs to be strengthened. Guidance on what tests are already available and prompt notice of any new tests (validated or non-validated) and how to incorporate them into testing strategies is needed at international and national levels and across sectors. Any such guidance must be relevant to optimize human safety. To make the guidance relevant, regulators need as much information as possible and, therefore, companies must show they understand the tests they apply and also present data in a clear but concise way. Ethics committees and national reference laboratories should receive up-to-date information frequently and promptly. Sharing of information, collaboration and harmonization between regulatory authorities, companies and experts need to become the norms. Education will be crucial to achieving change. Young scientists in universities and even in schools need to be aware of animal use and inspired to explore alternative methods.

\subsection{Validation time frames}

The time to validation can take years and the processes can be complicated, confusing and frustrating, which might deter companies from developing novel alternative methods. In Europe, the risk assessment process is done at two levels: one at the European Commission level and one at industry level. Companies must create dossiers on all the substances they use. These are passed to the responsible parties in the company (safety assessors) and the European Commission (Directorate General for Health \& Consumers) who must ensure the information is made clear to the public. The European Commission assigns substances to annexes - forbidden substances, restricted substances, colorants, preservatives and ultraviolet filters. Thus, the product information for all ingredients must be comprehensive to enable assignment to the correct annexes. Industry and the European Commission both use the same safety evaluation strategy: hazard identification, dose response for risk characterization and exposure assessment. Where this was traditionally done with in vivo tests, industry is now encouraged to take a 3Rs approach (replace and/or refine wherever possible to reduce the numbers of animals tested) and to review existing data from previous in vivo tests, from analogues and read-across studies. A major drawback that could be preventing greater degrees of innovation and of applicability, however, is that in vitro data generated for one industry frequently cannot be transferred for use by another. In the European Union, legislation for different industries (e.g., chemicals, food additives, cosmetics, pharmaceuticals and detergents) exists in parallel with little communication or collaboration. Additionally, having to work within OECD regulation guidelines removes the flexibility needed to accommodate product-related modifications. Thus, tests are duplicated for each industry and resubmitted for validation. At the same time, however, some "horizontal" legislation applies across all industries in all European Union countries, such as that on animal experimentation. To satisfy all requirements in this legislative structure can become incredibly difficult and requires a lot of excess time and effort. Increased communication and collaboration between industries and regulators would be an important step towards improving the validation process.

Application of methods after validation is another area of concern. The European Coalition to End Animal Experiments (ECEAE), a Europe-wide alliance, reported in the meeting that it has found that implementation of alternative methods is frequently held up after validation, which prevents quick and broad uptake. The alliance suggests that post-validation stages need to be taken into account during the process to speed and smooth the implementation of new tests. It summarizes the validation and post-validation stages as assessment, decision, acceptance, policing and transparency (ADAPT). The first hurdle is the decision about whether a novel approach needs to be tested before submission for validation (assessment) and is confused by a lack of clarity about which regulatory authority is the most suitable to assess a new approach and make the final decision that an alternative method is valid (decision). Reliance on international acceptance, for instance through further assessment by OECD, can substantially delay regional implementation and some methods are initially approved but face legal hold ups to implementation (acceptance). As suggested above, once methods are validated and accepted, it must be ensured that companies use them in preference to pre-existing animal tests (policing). Finally, the regulatory requirements and processes must be simplified and the frequency and effectiveness of collaborations and communication must increase (transparency).

\section{Innovations and potential solutions}

Although it became clear at the meeting that there are many hindrances to moving towards alternative methods, many positive points were raised about innovative thinking and possible ways to overcome hurdles that might be widely applicable. These ranged from ways to use existing data and how to record, organize and store new data, potential ways to increase the dissemination of informa- 
tion, improve information sharing and increase collaboration for models that have been developed to expand the range of substances that may be tested in vitro and state-of-the-art methods to analyze high throughputs of data and ways to address wider issues.

The Sneller van Innovatie naar Mens (SLIM), or Faster from Innovation to Man, project is an ongoing group project in the Netherlands that involves the government, pharmaceutical, food and other companies, academic institutions and various other partners. The objective is to identify good practices for smarter and faster development, acceptance (including regulatory) and implementation of $3 \mathrm{Rs}$ methods. The aim is to investigate how 3Rs approaches will fit better into current practices and processes, barriers to and drivers of regulatory acceptance and how industrial and regulatory needs can drive future goals. Four fields of research areas are being assessed: reproductive toxicity, food allergy, carcinogenicity and exposure barriers. These represent complicated areas of safety assessment for which the development of alternative methods is difficult (EFSA, 2009). Some of the non-animal methods that have proven helpful so far are mining of existing data and creation of comprehensive, widely accessible databases, use of in vivo repeated-dose toxicity studies to move away from 2-year rat carcinogenicity studies, use of human cell assays, use of high-throughput studies, and in vitro and in silico modeling of bioavailability. However, multiple factors - drivers as well as barriers - at different levels (individual, organization, institution, authority and society) influence innovation and need to be taken into account.

Repeatedly during the meeting, the issues came up of access to and dissemination of information. Rather than to tweak current approaches, a fundamental shift to "open source" information was suggested. This concept is widely understood by the IT community, has been utilized for around the past 30 years and has led to many innovations on varying scales, from small pieces of software to whole computer operating systems. To be deemed truly open source, a product and its intellectual property must be publically available, acceptable and readable, must not be subject to copyright or intellectual property protection, and elements may be altered by multiple developers to make the product fit for purpose. In any industry there are, of course, risks in making properties openly available. A particular issue in industries bound by safety analysis is whether regulation can be upheld if a product is made openly available after validation. That should not mean, however, that open source is wholly inapplicable to validated tests in Europe. It was suggested that exposure of products in the pre-validation stages could lead to optimized methods through the feedback of experts. In turn, the honed final product might be quicker and easier for regulators to approve. An adapted form of open source was suggested, in which tissue models and test performance data, but not the "source code" behind them, are made publically available with the right granted for others to produce and use those models in any way. This approach might help companies in restrictive shipping areas or small companies to access robust models by gaining the ability to make them on site. Of course, any tests and models would have to be developed to the highest standards and minimum requirements would need to be put in place and adherence proven. Any negative issues might, however, be outweighed by the benefits of a swift, wide dissemination of information (De Wever et al., submitted)
It was well recognized by meeting participants that the range of novel in vitro models and other methods will need to be broad to replace animal tests. Two innovative examples were presented. First was a three-dimensional airway epithelium model that is developed from nasal, bronchial, or tracheal epithelial cells that differentiate into all the relevant upper airway cells. This model is accompanied by a novel method to enable testing of non-water compatible substances. The substance is mixed with dextran in serial solid dilutions, compressed and the resulting tablet is applied to the epithelial model surface. Exposure is homogeneous across the surface of the model and the reactions are as they would be in vivo because of direct contact with the epithelium. Several endpoints may, therefore, be tested simultaneously. Currently, this model is used as part of an integrated testing strategy, the results are donor specific and limited numbers of cultures can be generated per donor (100-500 inserts), although a large pool of donors is overcoming this problem $(35,000$ inserts $)$. Although not yet stand-alone, this model illustrates how difficult substances can be dealt with in custom made models that have a long shelf life (approx. one year).

The next approach discussed was an imaging software package for which protocols may be adapted to suit the client's needs. Assessments can be fully automated and can improve accuracy, reproducibility, rate and cost-effectiveness of analysis. The software enables high-throughput analyses of individual elements within a sample. For instance, from an original red-green-blue image of a stained slide from the epithelial airway model, mucin and nuclei can be viewed separately (Fig. 1). Additionally, target regions can by highlighted by delineation or zoning (block coloring of the different elements in one image). This tool can help with the study of efficacy and toxicity and is widely applicable across the chemical, cosmetic, pharmaceutical and other industries.

Less innovative but just as important solutions are harmonization and standardization across countries, areas of regulation and industries. Such changes would greatly improve the regulatory process and the sharing of information and would reduce test duplication, effort and costs. In terms of test development, innovation will rank much higher than competition. For instance, as animal tests become phased out, it will be difficult to introduce competition for two similar tests. Unless there is a major innovative difference between them, the customer will be inclined to buy the cheaper one. Harmonization, therefore, could save not only end users' but also developers' time and money and encourage innovation and sharing of information. Again, a new approach is needed that moves away from company and product specificity. Standardization of terminology, test materials generation and delivery, methods of information storage and access will encourage integration of all stakeholders and international acceptance, which will in turn help with regulatory validation.

Finally, where alternative tests have been validated but the required tools are not yet readily available, some companies have designed their own instruments and made them available to other companies. Such activity has been undertaken by BASF with the development of the BCOP Opacitometer Kit BASF - OP3.0 (http://on.basf.com/1sNtuOZ) 


\section{Conclusions}

Partial replacement or refinement of methods as part of comprehensive testing strategies in order to reduce the number of animal tests has been possible, and some methods have been validated. In no area, however, can animal testing yet be completely replaced. Despite the time involved in development and validation, an overwhelming message to come out of the meeting was that it is better to try to create alternative methods that better predict the human situation than not to try at all. Mode-of-action and mechanistic studies to improve understanding of the pathways that lead to harm will play an important part in the required paradigm shift. Industry needs to start building strategies, initially on the basis of what tools are available right now, to help direct efficacy and safety questions, to adopt properly the alternative methods available and to move towards as little animal testing as possible. While there are animal protection laws in place that make animal tests a last resort, many hindrances, such as costs, shipping, expertise and so on mean that widespread implementation of sufficient alternative methods to replace them is slower than necessary. All stakeholders - industry, regulators, government, academia, animal welfare organizations and consumers - need, therefore, to not only promote the use of alternative tests, but also to co-ordinate to find pragmatic ways to help more companies implement them. The key to success will be an integrated approach that bridges the distance between science, policy, legislation and education to enable knowledge transfer. Companies will need to be persuaded that gains, such as improved prediction of effects, cost effectiveness and so on, can be made in the long run by not using animal models. In the broader arena, guidance on scientific issues related to safety should be sought from independent bodies, such as the Scientific Committee on Consumer Safety (SCCS), forums should be created for information sharing and exchange of ideas and solutions (safe harbor concept), and stakeholders should consider joining together to start dialogues about hindrances and other issues in an attempt to speed resolutions. Proactivity and collaboration to design and validate widely applicable, cost-effect alternative methods are urgently needed on all fronts.

\section{References}

Altenburger, R., Scholz, S., Schmitt-Jansen, M. et al. (2012). Mixture toxicity revisited from a toxicogenomic perspective. Environ Sci Technol 46, 2508-2522. http://dx.doi.org/10.1021/ es2038036

Ankley, G. T., Bennett, R. S., Erickson, R. J. et al. (2010). Adverse outcome pathways: A conceptual framework to support ecotoxicology research and risk assessment. Environ Toxicol Chem 29 , 730-741. http://dx.doi.org/10.1002/etc.34

CEC - Commission of the European Communities (2003). Commission staff working document: timetables for the phasing-out of animal testing in the framework of the $7^{\text {th }}$ amendment of the cosmetics directive (Council Directive 76/78/EEC). http://bit. ly/1or2nd5 (accessed 06.09. 2013).

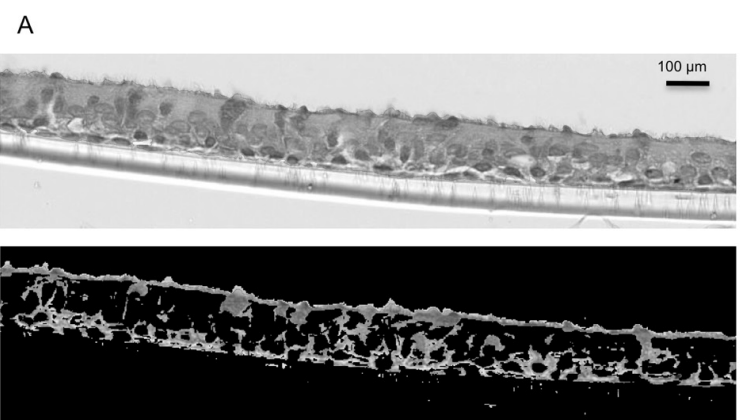

B
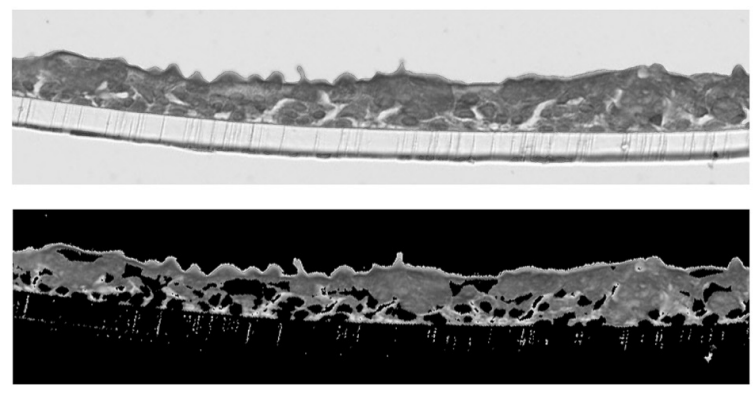

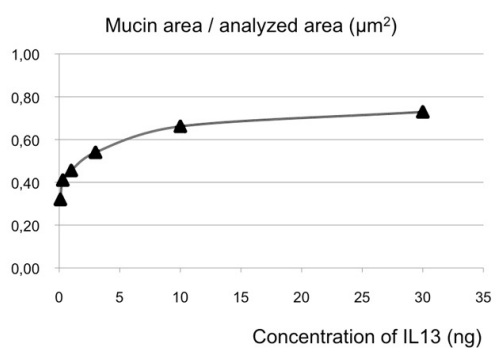

Fig. 1: Use of image-processing software to quantify mucin in goblet cells in a three-dimensional model of human bronchial epithelium

Mucin secretion was induced by an increased concentration gradient of IL-13 and sections from the model were stained with alcian blue. Discrimination processes were applied with the software to create images of the mucin component. Images of section (A) unaltered MucilAir (top) and after processing to show only the mucin component at $0.1 \mathrm{ng} / \mathrm{ml}$ (bottom) and (B) unaltered (top) and after processing to show only the mucin component at $30.0 \mathrm{ng} / \mathrm{ml}$ (bottom). All images ${ }^{\circledR}{ }^{B} \mathrm{BIOCELLVIA}$, Marseilles, France. 
Collings, F. B. and Vaidya, V. S. (2008). Novel technologies for the discovery and quantitation of biomarkers of toxicity. Toxicology 245, 167-174. http://dx.doi.org/10.1016/ j.tox.2007.11.020

Crump, K. S. (2011). Use of threshold and mode of action in risk assessment. Crit Rev Toxicol 41, 637-650. http://dx.doi. org/10.3109/10408444.2011.566258

De Wever, B., Goldberg, A., Eskes, C. et al. (2014). 'Open Source' based engineered human tissue models: A new gold standard for non-animal testing through openness, transparency and collaboration, promoted by the ALEXANDRA Association. Toxicol In Vitro, in press.

EC - European Commission (2010). Report from the commission to the Council and the European Parliament: Sixth Report on the statistics on the number of animals used for experimental and other scientific purposes in the Member States of the European Union SEC(2010) 1107. http://bit.ly/RQkGe3 (accessed 06.09. 2013).

EC (2013a). Communication from the Commission to the European Parliament and the Council on the animal testing and marketing ban and on the state of play in relation to alternative methods in the field of cosmetics. http://bit.ly/1mXM3ws (accessed 06.09. 2013).

EC (2013b). Commission staff working document: impact assessment of the animal testing provision in Regulation (EC) 1223/2009 on cosmetics. http://bit.ly/1nJ8klt (accessed 06.09. 2013).

EFSA - European Food Safety Authority (2009). Scientific Opinion of the Scientific Committee: "Existing approaches incorporating replacement, reduction and refinement of animal testing: applicability in food and feed risk assessment" (question no EFSA-Q-2005-231). EFSA J 1052, 1-77.

Ekin, S. (2006). Systems-ADME/Tox: Resources and network approaches. J Pharmacol Toxicol Methods 53,38-66. http://dx.doi. org/10.1016/j.vascn.2005.05.005

EU (2010). Directive 2010/63/EU of the European Parliament and the Council of 22 September 2010 on the protection of animals used for scientific purposes. http://bit.ly/1sNtcI7 (accessed 06.09. 2013).

Hardy, B., Apic, G., Carthew, P. et al. (2012). Toxicology ontology perspectives. ALTEX 29, 139-156. http://dx.doi.org/10.14573/ altex.2012.2.139

Hill, A. B. (1965). The environment and disease: association or causation? Proc R Soc Med 58, 295-300. http://www.ncbi.nlm. nih.gov/pmc/articles/PMC1898525/ (accessed 14.04.2014).

Leist, M., Hartung, T., and Nicotera, P. (2008). The dawning of a new age of toxicology. ALTEX 25, 103-114. http://www.altex.ch/ All-issues/Issue. 50. html?iid=99\&aid=3 (accessed 14. 04.2014).

OECD - Organisation for Economic Co-operation and Development (2013). Guidance document on developing and assessing adverse outcome pathways: series on testing and assessment no. 184. http://bit.ly/1nNITN7 (accessed 06.09. 2013).

Panagiotou, G. and Taboureau, O. (2012). The impact of network biology in pharmacology and toxicology. SAR QSAR Environ Res 23, 221-235. http://dx.doi.org/10.1080/1062936X.2012. 657237

Rebholz-Schuhmann, D., Oellrich, A. and Hoehndorf, R. (2012). Text-mining solutions for biomedical research: Enabling in- tegrative biology. Nat Rev Genet 13, 829-839. http://dx.doi. org/10.1038/nrg3337

Schrage, A., Hempel, K., Schulz, M. et al. (2011). Refinement and reduction of acute oral toxicity testing: A critical review of the use of cytotoxicity data. Altern Lab Anim 39, 273-295. http://bit. ly/1or2khp (accessed 14.04.2014).

Zuang, V., Schäffer, M., Tuomainen, A. M. et al. (2013). JRC scientific and policy reports: EURL ECVAM progress report on the development, validation and regulatory acceptance of alternative methods (2010-2013). Luxembourg: Publications Office of the European Union, 2013.

\section{Conflicts of interest}

The authors declare they have no conflicts of interest.

\section{Acknowledgements}

Speakers: Herman Koëter, Orange House Partnerhsip, Belgium; Thomas Hartung, CAAT, USA; Elisabeth Berggren, EURL-ECVAM, Italy; Erwin Roggen, Novozymes AS, Denmark; Marianna Gaca, British American Tobacco, UK; Albrecht Poth, Harlan, Germany; Cyrille Krul, TNO, Netherlands; David Basketter, DABMED Consultancy, UK; Kate Willet, Humane Society of the United States, USA; Karsten Mewes, Henkel, Germany; Samuel Constant, Epithelix, Switzerland; Yvon Jule, Biocellvia, France; Katy Taylor, ECEAE/BUAV, UK; Vera Rogiers, Scientific Commission of Consumer Safety (SCCS), European Commission, Brussels, Belgium; Tzutzuy Ramirez, BASF, Germany; Jos Bessems, RIVM, The Netherlands.

IVTIP members: Ahava - Dead Sea Laboratories, Israel; Alcyomics Ltd, UK; Beiersdorf AG, Germany; Biocellvia, France; BioDetection Systems bv, The Netherlands; BioMed-zet Life Science GmbH, Austria; Biopredic, France; Biopta Ltd, UK; BioTeSys GmbH, Germany; British American Tobacco, UK; BSL Bioservice, Germany; CeeTox, USA; CellSystems GmbH, Germany; Citoxlab, France; Epithelix, Switzerland; Evocutis plc, UK; Fraunhofer IGB, Germany; Gaiker, Spain; Harlan Laboratories Ltd, UK; Henkel, Germany; IIVS - Institute for In Vitro Sciences, USA; InSphero AG, Switzerland; KaLy-Cell, France; Kallistem, France; Kirkstall Ltd, UK; Leitat, Spain; Leopharma, Denmark; L'Oréal, France; MB Research Labs, USA; Medicyte GmbH, Germany; Natura, France/Brazil; Novozymes A/S, Denmark; Novo Nordisk A/S, Denmark; Philip Morris Products SA, Switzerland; Readycell, Spain; Reinnervate, UK; Scantox, Denmark; Sterlab, France; Thor Personal Care SAS, France; TNO Quality of Life, The Netherlands; Tridskin, Brazil; Vitrocell Systems GmbH, Germany

\section{Correspondence to}

Bart De Wever, $\mathrm{PhD}$

ALTEXA Development, Columbia Palace, 11

Avenue Princesse Grace

Monte Carlo 98000, Monaco

Phone: +33687214068

e-mail: bart@deweverconsulting.be

http://www.altexa.net 